(C2019 IEEE. This is the authors version of the work. It is posted here for your personal use. Not for redistribution. The definitive Version of Record was published in Proceedings of the 2019 Design, Automation \& Test in Europe Conference \& Exhibition (DATE 2019), Florence, Italy, 2019, pp. 872-877.

Personal use of this material is permitted. Permission from IEEE must be obtained for all other uses, in any current or future media, including reprinting / republishing this material for advertising or promotional purposes, creating new collective works, for resale or redistribution to servers or lists, or reuse of any copyrighted component of this work in other works.

DOI: $10.23919 /$ DATE.2019.8715181 


\title{
Cost/Privacy Co-optimization in Smart Energy Grids
}

\author{
Alma Pröbstl, Sangyoung Park, Sebastian Steinhorst, Samarjit Chakraborty \\ Technische Universität München, Germany \\ \{alma.proebstl, sangyoung.park, sebastian.steinhorst, samarjit.chakraborty\}@tum.de
}

\begin{abstract}
The smart energy grid features real-time monitoring of electricity usage such that it can control the generation and distribution of electricity as well as utilize dynamic pricing in response to the demands. For this purpose, smart metering systems continuously monitor the electricity usage of customers, and report it back to the Utility Provider (UP). This raises privacy concerns regarding the undesired exposure of human activity and time-of-use of home appliances. Photovoltaics (PV) and a residential Electrical Energy Storage (EES) have proven to be effective in mitigating the privacy concerns. However, this comes at several costs: Installation of PV and EES, their subsequent aging and the possibly increased electricity cost. We quantify the trade-off between privacy exposure and financial costs by formulating a stochastic dynamic programming problem. Our analysis shows that $i$ ) there is a quantifiable trade-off between the financial cost and privacy leakage, ii) proper control of the system is crucial for both metrics, iii) a strategy solely focusing on privacy results in high financial costs, and iv) that for a typical residential setting, the costs for a trade-off solution lie in the range of $600 \mathrm{US} \$-1700 \mathrm{US} \$$. As the load flattening has a peak shaving effect desirable for UPs, increasing privacy is mutually beneficial for both, customers and UPs.
\end{abstract}

\section{INTRODUCTION}

Smart grids promise more efficient electricity generation and distribution thanks to the use of information and communication technologies. Dynamic energy pricing motivates the users to shift loads and perform demand side management increasing the energy efficiency of the grid. All this is enabled by the use of smart meters, which continuously monitor the load and communicate the data to the Utility Provider (UP). The UP can then set electricity prices to encourage customers to voluntarily shift the load out of the peak hours. At grid scale, the avoidance of peak loads increases load predictability and reduces the need for costly fossil-fuel reserve generators, which enhances the energy efficiency and reduces the carbon dioxide emission.

Privacy leakage by smart meters: The load profiles gathered by smart meters convey private information [1], [2]. While conventional electricity meters only record the accumulated usage over a month, smart meters allow to access real-time, fine-grained data. In the worst case, if accessed by an unauthorized third party, information on residential activities can be extracted. E.g., Non-Intrusive Load Monitoring (NILM) could be used to identify individual appliances and usage profiles [3] Prior works: Prior works have aimed at reducing the privacy leakage by using a battery storage to modify and hide the usage pattern of appliances and human activities [4]-[7]. Other works propose distributed load shifting [8]. Also, the impact of

With the support of the Technische Universität München - Institute for Advanced Study, funded by the German Excellence Initiative and the European Union Seventh Framework Programme under grant agreement no. 291763 And with the support of the German Federal Ministry for Education and Research with grant agreement no. 01DQ17018 (FutureTrans).

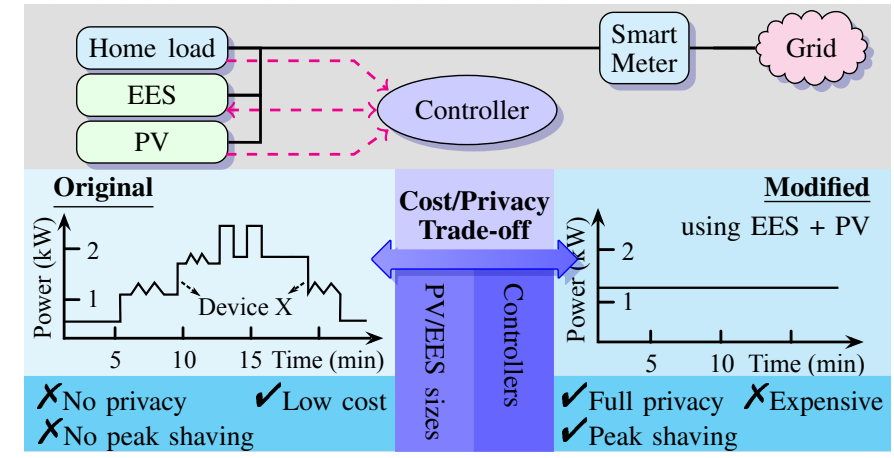

Fig. 1. The original profile seen at the smart meter can be modified through a water filling strategy using EES and PV to increase privacy at increased cost. We propose a cost/privacy trade-off.

additional renewable energies on the privacy leakage rate has been investigated [7]. Generally, the proposed algorithms either flatten [4], [5] or randomize the load [8]. Interestingly, these works overlooked an important advantage of load flattening: Its peak shaving effect is very much desirable for the UP's demand side management by increasing the predictability of grid power and hence reducing reserve power. Thus, load flattening is mutually beneficial for customers as well as UPs.

While the effectiveness of the approaches in privacy protection has been well studied, there has been limited research on its cost. There have been approaches to reduce battery energy losses [4], electricity cost of a dynamic pricing policy [5] and maintenance cost in terms of battery cycle life [4], [5]. However, the latter is one of the most important factors and has not been considered properly, mainly due to the complexity of the battery aging models. Previous work calculates the battery degradation based on cycle counting [5], which is very inaccurate unless very simplistic battery usage profiles are assumed. Other works consider the effect of Depth of Discharge (DOD) on battery aging, but neglect other important factors such as average State of Charge (SOC) and C-rate [4], where C-rate is defined as the battery current relative to the battery capacity. However, it is widely known that the battery aging not only depends on the number of charge/discharge cycles or DOD, but also on other factors such as average SOC, temperature and C-rate. As such works intend to develop sophisticated battery management policies resulting in complex battery usage, cycle life estimation will be inaccurate without considering such factors, and proper analysis on the relationship between the privacy enhancement and its costs cannot be made. Today, battery costs are significant and proper estimation is necessary to evaluate the cost of privacy protection.

Trade-off between privacy and cost: The general setup is shown in Figure 1, An EES and PV jointly modify the 
power profile such that less residential activity information can be extracted. The power generated by the PV could be either used directly to satisfy any household appliances or it could be stored in the EES. Similarly, electricity could be drawn from the grid and be stored in the EES for later use. One strategy for privacy protection would be the water filling policy [4], which completely flattens the load profile and leaks no privacy information, see Figure 1 . This particular strategy has the additional advantage of being beneficial for the UP's demand side management through its peak shaving effect, a synergy that should be exploited. On the other hand, costs arise from PV and EES installation and maintenance and possibly increased electricity bills. E.g., larger EES sizes provide more flexibility for privacy protection, but come at a higher cost. Larger PV sizes are financially beneficial by reducing the use of grid electricity, but their usefulness in protecting privacy should be carefully evaluated as, if too large, they might saturate the EES and reduce its capability of flattening the load profile. Apart from that, EES degradation depends very much on the usage pattern, i.e., the chosen control actions. In general, higher EES usage increases the aging rate.

Proposed work: In this paper, we, for the first time, perform a comprehensive analysis on the trade-off between the privacy enhancement with concurrent peak shaving and the associated financial overhead. To the best of our knowledge, this is the first work to present a control strategy that not only minimizes the privacy leakage and performs peak shaving but also minimizes the financial cost with realistic battery aging models. In order to jointly consider the reduction of privacy leakage and financial costs, we synthesize an optimal controller for a given EES and PV setup. We model a whole residential system as a Markov Decision Process (MDP), formulate an average reward maximization problem, and derive the optimal controller using a relative value iteration algorithm.

The contributions of this paper are summarized as follows.

- We propose a control strategy for a system consisting of EES and PV that minimizes both financial cost, i.e., the sum of battery and PV depreciation and grid electricity costs, as well as the privacy enhancement, that simultaneously results in peak shaving.

- We quantify the trade-off of privacy enhancement with peak shaving and financial overhead under privacy and financial cost optimal control strategies. Using our framework, we identify the Pareto-optimal solutions.

- We achieve increased accuracy in our solution and analysis by using an elaborate battery model in our framework that is more precise compared to models in previous works and considers SOC, C-rate and temperature.

- We identify balanced strategies that trade-off privacy and financial cost. Towards this, we show that, while full privacy can be achieved at acceptable but non-negligible costs, a controlled cost/privacy trade-off based on our methodology reduces these costs by more than half.

- Due to the correlation of privacy enhancement and peak shaving, which mutually benefit consumer and UP, we propose cost splitting of trade-off-strategies, potentially creating a new business model.

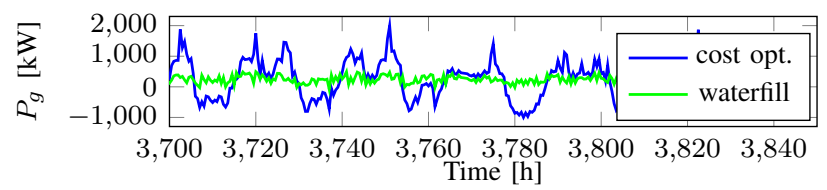

Fig. 2. Grid power of privacy focused water filling $w_{p}=1$ vs. grid cost focused strategy $w_{g}=1$. The mutual information for the privacy and grid cost focused strategy is 10.733 and 11.28, respectively. Data: EES 3 and PV 1 , June 4-10, 2015.

The paper is organized as follows. Section II reviews privacy concerns and shows the problem formulation. System modeling and controller derivation are found in Sections III and IV respectively. Simulation results are discussed in Section V

\section{Motivational Example and Problem Statement}

A number of algorithms exist for extracting privacy information from an electricity usage trace. Among them, NILM algorithms are capable of identifying when individual appliances are turned on and off [9], and of distinguishing different instances of light bulbs using cluster analysis [10]. Such information could be exploited by malicious attackers to find absence routines of the residents and to disclose their behaviors. One possibility for full privacy protection would be a water filling strategy such as the one shown in Figure 1 using EES and PV. The EES is used to hide the residential load completely such that the power usage seen from the grid is almost constant and maintained around a target line. On the downside, this strategy would extensively use the EES and not optimize towards time-of-use prices resulting in accelerated EES degradation in addition to increased electricity cost. In Figure 2, we show two grid power profiles of a home equipped with PV and EES resulting from a financial cost focused strategy and a privacy focused strategy. In our results, we find that the total system cost of privacy leakage reduction and peak shaving sums up to around 1300 to 1600 US\$ per year, while a conventional, financial cost optimal set-up reduces the electricity bill and costs around 600 US\$ per year. This high discrepancy, which is dominated by battery aging costs, confirms the need for an improved cost analysis by using a more accurate battery aging model than the ones in previous works [4], [5]. A user wanting some privacy protection would desire a balancing of cost and privacy. Our optimization objective therefore is to minimize privacy leakage, battery aging and grid cost at the same time. A bilateral profit originates from load profile flattening also being known as peak shaving, which benefits the UPs' demand side management and motivates cost splitting among the involved parties.

Problem statement: We propose to solve this multiobjective optimization problem by formulating a singleobjective weighted sum problem. The weighted objective consists of the cost associated to buying and selling electricity to and from the grid $c_{g}$, the battery degradation cost $c_{h}$ and the privacy cost $c_{p}$, here determined by the deviation of the grid power from the average household load power. We want to find the EES current $I_{\mathrm{EES}}(t)$ that minimizes the sum of the aforementioned costs over the total lifetime of the system:

$\min _{I_{\mathrm{EES}}} \lim _{T \rightarrow \infty} \sum_{t=0}^{T} w_{g} c_{g}\left(t, I_{\mathrm{EES}}\right)+w_{h} c_{h}\left(t, I_{\mathrm{EES}}\right)+w_{p} c_{p}\left(t, I_{\mathrm{EES}}\right)$, 


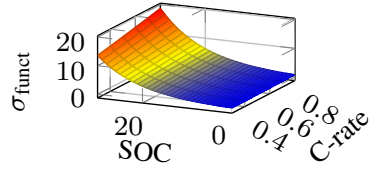

Fig. 3. Severity factor map for $25^{\circ} \mathrm{C}$.

with $w_{g}, w_{h}$ and $w_{p}$ being the associated weights. We need a decision at each time instance $t$ and reformulate the objective:

$$
\forall t: \min _{I_{\mathrm{EES}}} w_{g} c_{g}\left(t, I_{\mathrm{EES}}\right)+w_{h} c_{h}\left(t, I_{\mathrm{EES}}\right)+w_{p} c_{p}\left(t, I_{\mathrm{EES}}\right)
$$

\section{SySTEM MOdELS}

In this section, we present the models required in the MDP optimization framework: An elaborate battery cycle life model, which is crucial for correctly assessing the financial cost, a stochastic residential load model, a PV power generation model, a dynamic energy pricing policy and a privacy metric.

\section{A. Battery Cycle Life and Associated Cost}

We model the Li-ion battery behavior according to the equivalent circuit model in [11] and derive its cycle life degradation in terms of an empirically fitted severity factor map [12]. The severity factor $\sigma_{\text {funct }}$ describes the amount of damage done to a battery by its current SOC, the applied C-rate and the temperature as shown in Figure 3 This cycle life model is particularly developed for control applications as the computational effort is comparably low and no iterative dependencies exist. The model is derived for Li-ion batteries, which are commonly used in hybrid electric vehicles but also in stationary storages. The framework can be easily adopted to other cell chemistries by data fitting as in [13].

We use the severity factor $\sigma_{\text {funct }}$ for the controller design and determine the financial loss due to EES degradation. Assuming that a new storage is purchased at some cost $C_{\mathrm{EES}}$ in US\$ and that an EES that has reached its End of Life (EOL), i.e., when $80 \%$ of the initial capacity is left, is worth 0 US\$, we calculate the cost in US\$ in dependence of the capacity loss $Q_{\text {loss,\%: }}$ :

$$
c_{\mathrm{SOH}}=\frac{1}{1-\mathrm{EOL}} C_{\mathrm{EES}} Q_{\mathrm{loss}, \%}
$$

\section{B. Residential Load Model}

We use the publicly available UMass Smart* Home Data Set [14] to model a residential load. The data set monitors several appliances in a home with three residents. The accumulated load power of Home A in 2014 is used for model training and in order to show the effectiveness of the method, other data than the training data is used for the evaluation, i.e., Home A data from 2015. Most times, the residential load data is in the range of $0 \mathrm{~kW}$ to $2 \mathrm{~kW}$. Rarely, higher values occur, and in a preprocessing step these data points are removed in order to reduce the state space of our model and simulation time. We use six equally spaced power states whose probabilities are shown in Figure 4 We compute the transition probability matrix $M_{\mathrm{Ld}}$ from the transition histogram for Home A in 2014

\section{PV Power Generation Model and PV Cost}

PV Power Generation: The PV power generation depends on the location and orientation of the PV cells as well as the solar irradiance, which varies according to the cloud cover. If more clouds occlude the sky, the irradiance is less and therefore less PV power is produced. We use the cloud cover from the data set [14] we also used for the load model and calculate the cloud cover transition probability matrix. We use the model from [15] to then derive the solar irradiance. The model requires the latitude and longitude associated to the cloud cover data. Due to data protection, the exact location is not conveyed in the data set, but the rough location is known to be in Western Massachusetts [16]. As any location within that region suits our purposes, we choose the latitude $42.45^{\circ}$ and longitude $-73.2458333^{\circ}$ of Pittsfield. The solar irradiance is then integrated into a PV model [17]. We incorporate the dominant PV characteristics, but refining might be possible by considering further factors, such as series-parallel connections of panels, shading, bypass diodes and choice of microinverters. PV Cost: Additionally to the gain from PV power selling, costs of PV installation and degradation occur. We assume a cost of $1.37 \mathrm{US} \$ / \mathrm{W}$ in 2011 [18] and an overall installation cost including other factors such as the inverter of $3.43 \mathrm{US} \$ / \mathrm{W}$ for rooftop use. The PV degradation rate can be estimated to be $0.8 \%$ per year [19]. The EOL is reached at $80 \%$ degradation or after a lifetime of 25 years with linear degradation.

\section{Dynamic Energy Pricing}

Modern grid energy price policies vary the prices depending on the time of the day, such as the one offered by the Los Angeles Department of Water and Power [20]. Below are the prices in US\$ per $\mathrm{kWh}$ for weekdays in June 2017.

$$
c_{\text {USD }}(t)= \begin{cases}0.13967 \text { US\$ } & \text { for 00:00-09:59, 20:00-23:59 } \\ 0.16411 \text { US\$ } & \text { for 10:00-12:59, 17:00- 19:59 } \\ 0.24328 \mathrm{US} \$ & \text { for 13:00-16:59 }\end{cases}
$$

Selling energy comes at a gain of 0.145 US\$ per $\mathrm{kW} \mathrm{h} \mathrm{[20].}$

\section{E. Mutual information}

We use mutual information as a metric to quantify the privacy exposure as in many prior works [6], [7]. Mutual information $I(X ; Y)$ is an information theoretic quantification metric that describes the amount of information that one random variable $X=\left\{x_{1}, x_{2}, \ldots, x_{n}\right\}$ contains about another random variable $Y=\left\{y_{1}, y_{2}, \ldots, y_{n}\right\}$. It is defined as

$$
\begin{aligned}
I(X ; Y) & =H(X)+H(Y)-H(X, Y) \\
& =\sum_{x \in X} \sum_{y \in Y} p(x, y) \log _{2} \frac{p(x, y)}{p(x) p(y)} .
\end{aligned}
$$

The average amount of information contained in one random variable is expressed by its entropy, $H(X)$ and $H(Y)$, and $H(X, Y)$ denotes the joint entropy. Here, the two random variables are the original residential grid power consumption and the new grid power draw after the original load has been modified using EES and PV. We use the mutual information to evaluate the performance of our control strategies.

\section{Financial Cost and Privacy Optimization}

To solve the optimization problem stated in Section II, we formulate an MDP average reward maximization problem based on the component models from the previous section. The goal is to minimize the weighted sum of electricity cost, battery depreciation cost, and privacy exposure. We provide the transition probability matrix (TPM), transition reward matrix (TRM) and synthesize a controller. 
States, actions and reward: An MDP enhances Markov chains by actions and rewards. The latter ones motivate state transitions as the goal is to maximize the reward. Actions and random variables influence the transitions between states. In our problem, a state $b \in \mathcal{B}$ is defined by the tuple $(c c, z, l, s)$ consisting of the present cloud cover $c c \in \mathcal{C C}$ at a given timeof-day $z \in \mathcal{Z}$, resulting in a certain PV power, the load power $l \in \mathcal{L}$ and the EES SOC $s \in \mathcal{S}$. A change in any of the four variables results in a state transition. Changes in time-of-day are deterministic as the next time-of-day state depends on the previous one. The action $a \in \mathcal{A}$ is the EES charge or discharge current. A non-zero EES current automatically results in a state change. The reward $R\left(b, b^{\prime}\right)$ for transitioning from one state $b$ to another state $b^{\prime}$ takes values in $r \in \mathcal{R}$. The overall reward depends on $z$ due to the time-of-use pricing scheme and is the weighted sum of privacy leakage, aging cost and the cost from buying or selling electricity to or from the grid.

Transition Probability Matrix: The TPM contains the probability for a transition from state $b$ to state $b^{\prime}$ if action $a$ is taken. Assuming that the cloud cover and load are independent processes, we can simply multiply the respective probabilities: $\operatorname{Pr}_{a}\left(b, b^{\prime}\right)=\operatorname{Pr}_{c c}\left(c c_{b}, c c_{b}^{\prime}\right) \operatorname{Pr}_{z}\left(z_{b}, z_{b}^{\prime}\right) \operatorname{Pr}_{l}\left(l_{b}, l_{b}^{\prime}\right) \operatorname{Pr}_{s, a}\left(s_{b}, s_{b}^{\prime}\right)$

The sequence of states is aligned with time and the probability of moving from one time-of-day to the subsequent one is $\operatorname{Pr}_{z}\left(z_{b}, z_{b}^{\prime}\right)=1$, while all other transitions of $z$ are 0 . Contrary to the probabilities of cloud cover transition $\operatorname{Pr}_{c c}\left(c c_{b}, c c_{b}^{\prime}\right)$ and load power transitions $\operatorname{Pr}_{l}\left(l_{b}, l_{b}^{\prime}\right)$, the SOC transition probabilities $\operatorname{Pr}_{s, a}\left(s_{b}, s_{b}^{\prime}\right)$ depend on the action $a$. The SOC change is largely deterministic, but the accuracy of run-time estimation of SOC is known to be limited, and hence, we probabilistically model the transition. We assume that the probability of the actual SOC is uniformly distributed around the estimate $s_{b}$ from $s_{b}-s_{u} / 2$ to $s_{b}+s_{u} / 2$, where $s_{u}$ is the SOC granularity. Hence, the probability of the actual SOC after $\Delta t$, would be uniformly distributed in a window of length $s_{u}$ around $s_{b}+I_{c}(a) \Delta t / 3600$ where $I_{c}(a)$ is the C-rate corresponding to $a$. We omit the exact equation for $\operatorname{Pr}_{s, a}\left(s_{b}, s_{b}^{\prime}\right)$ due to space constraints.

Transition Reward Matrix: The objective of the problem is to find a policy $\pi(b)$ that defines an action $a$ to be taken when in state $b$. The average reward over an infinite time window should be maximized:

$$
\lim _{T \rightarrow \infty} E\left[\sum_{t=0}^{T} R_{a}\left(b_{t}\right)\right],
$$

where $R_{a}\left(b_{t}\right)$ is the instantaneous reward with respect to state $b_{t}$ at time $t$, and $a$ is the action to be taken when in $b_{t}$. Here, the instantaneous reward $R_{a}\left(b, b^{\prime}\right)$ when transitioning from state $b$ to $b^{\prime}$ is the weighted sum of the inverse normalized costs for battery depreciation $c_{h \text {,norm }}$, grid related expenses from buying or selling electricity $c_{g \text {,norm }}$ and action $a$ induced privacy leakage $c_{p \text {,norm }}$ :

$$
\begin{aligned}
R_{a}\left(b, b^{\prime}\right)= & w_{g}\left(1-c_{g, \text { norm }}\left(b, b^{\prime}\right)\right)+w_{h}\left(1-c_{h, \text { norm }}\left(b, b^{\prime}\right)\right) \\
& +w_{p}\left(1-c_{p, \text { norm }}\left(b, b^{\prime}\right)\right)
\end{aligned}
$$

where the $w_{g}, w_{h}$, and $w_{p}$ are the respective weights. Due to normalization, we let the sum of the weights equal 1 .
TABLE I

MAXIMUM AND MINIMUM COSTS

\begin{tabular}{lll}
\hline $\mathbf{j}$ & $\mathbf{c}_{\mathbf{j}, \max }$ & \\
\hline$h$ & $\sigma_{\text {funct }}\left(1, I_{c, \max }, \theta_{\mathrm{EES}}\right)$ & $100 \%$ SOC, highest possible C-rate \\
$g$ & $P_{g, \max } \Delta t c_{\mathrm{USD}}$, peak & max. grid power $P_{g, \max }$ at peak hours \\
$p$ & $\left|P_{g, \max }-\rho\right|$. & max. grid power \\
\hline $\mathbf{j}$ & $\mathbf{c}_{\mathbf{j}, \min }$ & \\
\hline$h$ & $\sigma_{\text {funct }}\left(0,0, \theta_{\mathrm{EES}}\right)$ & $0 \%$ SOC, zero discharge current \\
$g$ & $-P_{g, \max } \Delta t c_{\mathrm{USD}, \text { peak }}$ & max. grid selling of PV and EES power \\
$p$ & $c_{p, \max }=0$. & no diversion from target value \\
\hline
\end{tabular}

Battery aging, grid and privacy cost: The cycle life cost is determined by the severity factor for the given SOC $s_{b}$, the C-rate stemming from action $a$ and the temperature $\theta_{\mathrm{EES}}$. Assuming a perfect cooling, the latter is constant.

$$
c_{h}\left(b, b^{\prime}\right)=f\left(s_{b}, a\right)=\sigma_{\text {funct }}\left(s_{b}, I_{c}(a), \theta_{\mathrm{EES}}\right)
$$

The grid cost is determined by the amount of power drawn from the grid $P_{g}$, which can easily be derived from the required load power, the cloud cover dependent PV generation and the EES power using $P_{\mathrm{EES}}=P_{\text {load }}-P_{\mathrm{PV}}-P_{\mathrm{g}}$. The grid electricity cost at some time-of-day is applied for period $\Delta t$.

$$
c_{g}\left(b, b^{\prime}\right)=f(a, c c, z, l)=P_{g}(a, c c, l) \Delta t c_{\mathrm{USD}}(z)
$$

Finally, we model the privacy cost as the diversion of the grid power $P_{g}$ from a defined target value $\rho$. From an information theoretic perspective, such flattening of the power profile effectively reduces mutual information and simultaneously achieves peak shaving.

$$
c_{p}\left(b, b^{\prime}\right)=f(a, c c, z, l)=\left|P_{g}(a, c c, l, z)-\rho\right|
$$

The target value $\rho$ equals the average load power - which can be estimated from past values, i.e., the average load over a year - reduced by the average PV generation. Even though the PV generation is subject to high seasonal as well as daily variations, in a real system, it can be estimated for a particular day from weather forecasts. We synthesize different controllers for discretized levels of PV generation. Above costs are normalized using max. and min. cost from Table $\mathrm{I}$.

$$
c_{j, \text { norm }}\left(b, b^{\prime}\right)=\frac{c_{j}\left(b, b^{\prime}\right)-c_{j, \text { min }}}{\left|c_{j, \text { max }}-c_{j, \text { min }}\right|}, \quad j \in\{g, h, p\}
$$

Solution: The MDPToolbox from [21], that is used to solve our optimization problem in MATLAB, implements a relative value iteration algorithm to find the controller which maximizes the long term average reward. The interested reader may refer to [22] to get more information on the algorithm.

\section{Simulation Results}

The evaluation of the controllers derived above is done on a custom developed simulator in MATLAB that also considers converter efficiencies for connecting EES and PV to the grid. We compare three EES and three PV sizes, as shown in Table II and vary the weights of the cost function. The sizes of EES 2 and 3 are the two smallest commercially available Tesla Powerwall storages [23]. EES 1 has half the capacity of EES 2. The sampling time $\Delta t=1800 \mathrm{~s}$ is the same as the one used in the load data set and is also typical for smart meters. The number of discrete states for EES SOC $S$, cloud cover $C C$ and load power $L$ is 21,9 and 6 , respectively. The number of discrete actions, i.e., the number of discrete EES current levels, depends on the EES size and is 51, 126 and 251 for EES 1, 2 and 3, respectively. The EES is maintained at $\theta=25^{\circ} \mathrm{C}$, the initial SOC is $S_{\text {init }}=0.5$ and the 
TABLE II

PARAMETRIZATION FOR EES AND PV SIZES

\begin{tabular}{llll}
\hline parameter & EES1 & EES2 & EES3 \\
\hline nominal size & $6.75 \mathrm{kWh}$ & $13.5 \mathrm{kWh}$ & $27 \mathrm{kWh}$ \\
purchase cost & $5 \mathrm{k} \mathrm{US} \$$ & $8 \mathrm{k} \mathrm{US} \$$ & $15.4 \mathrm{k} \mathrm{US} \$$ \\
$P_{\mathrm{ESS}, \min }, P_{\mathrm{ESS}, \max }$ & $\pm 2.3 \mathrm{~kW}$ & $\pm 4.59 \mathrm{~kW}$ & $\pm 9.18 \mathrm{~kW}$ \\
$P_{\mathrm{g}, \max }$ & $4.3 \mathrm{~kW}$ & $6.59 \mathrm{~kW}$ & $11.18 \mathrm{~kW}$ \\
$\#$ actions $a$ & 51 & 126 & 251 \\
\hline parameter & PV1 & PV2 & PV3 \\
\hline$P_{\mathrm{PV}, \text { out }}$ & $1 \mathrm{~kW}$ & $3 \mathrm{~kW}$ & $5 \mathrm{~kW}$ \\
purchase cost & $3430 \mathrm{US} \$$ & $10290 \mathrm{US} \$$ & $17150 \mathrm{US} \$$ \\
\hline
\end{tabular}

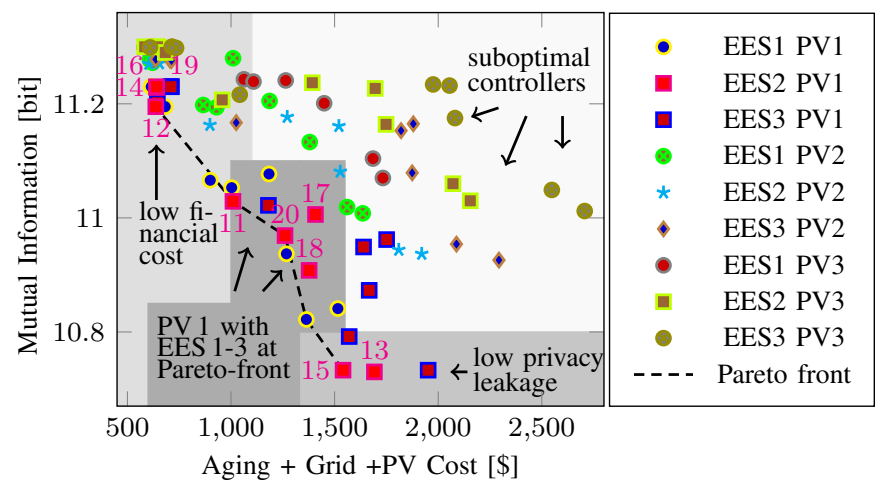

Fig. 5. DSE: Trade-off between privacy (low mutual information) and the total cost of ownership for EES and PV combinations and different strategies. The selected node labels link to the indices in Table [II

$\mathrm{EOL}=0.8$. We find that the grid power target level $\rho$ should vary depending on the predicted PV power generation on a particular day. It changes based on the weather prediction and has a discretization granularity of $0.25 \mathrm{~kW}$ steps. The predictor errors are acceptable as the can easily be compensated.

Privacy-cost trade-off: We are interested in the Paretooptimal settings in terms of EES size, PV size and weights. Figure 5 shows the financial cost versus the privacy leakage, which is equivalent to the degree of peak shaving. Different data points within a data group, defined by PV and EES size combinations, denote results for different weight values. As expected, higher weights for a particular reward come with better performance for the reward, but a worse one for other rewards. Strategies with high grid weight generally show lower cost but higher privacy leakage. Strategies with high privacy weight generally result in higher cost but lower privacy leakage. However, the gain in one domain is not always linear with the loss in another domain and hence a DSE approach is applied to ensure the best quality of control achieved at the lowest financial cost. The Pareto-front is depicted as a dashed line. We can observe a trade-off relationship between the financial cost and the privacy leakage. The spread of mutual information may seem small at first. But the grid power profiles of this seemingly low difference actually differ very much. As shown in Figure 2 the grid power profile for a privacy-optimal strategy, $w_{p}=1$, with mutual information 10.733 is relatively flat, while the profile for a grid cost optimal strategy, $w_{g}=1$, with mutual information 11.28 has much higher variation. The controllers synthesized with a particular combination of weight values, namely the ones whose results are part of the Paretofront, perform better even though the EES and PV sizes are same. This shows the significance of our approach: the quality
TABLE III

DSE SELECTED RESULTS - PV 1, EES 2

\begin{tabular}{rrrrrrrr}
\hline idx & EES [kWh] & PV $[\mathrm{kW}]$ & $w_{g}$ & $w_{h}$ & $w_{p}$ & Fin. Cost & mutInf \\
\hline 11 & 13.5 & 1 & $\frac{1}{3}$ & $\frac{1}{3}$ & $\frac{1}{3}$ & 1009.30 & 11.029 \\
12 & 13.5 & 1 & 1 & 0 & 0 & 637.05 & 11.195 \\
13 & 13.5 & 1 & 0 & 0 & 1 & 1692.00 & 10.730 \\
14 & 13.5 & 1 & 0 & 1 & 0 & 641.72 & 11.229 \\
15 & 13.5 & 1 & 0.5 & 0 & 0.5 & 1540.70 & 10.733 \\
16 & 13.5 & 1 & 0.5 & 0.5 & 0 & 641.72 & 11.229 \\
17 & 13.5 & 1 & 0 & 0.5 & 0.5 & 1407.60 & 11.006 \\
18 & 13.5 & 1 & 0.4 & 0.2 & 0.4 & 1377.00 & 10.908 \\
19 & 13.5 & 1 & 0.4 & 0.4 & 0.2 & 641.10 & 11.230 \\
20 & 13.5 & 1 & 0.2 & 0.4 & 0.4 & 1260.70 & 10.969 \\
\hline
\end{tabular}

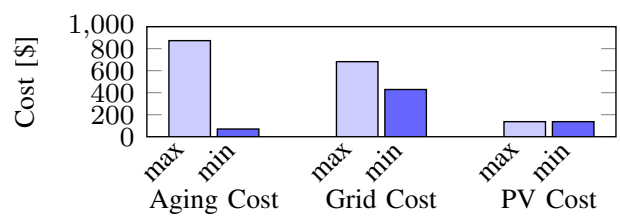

Fig. 6. Cost breakdown EES 2, PV 1: Aging cost varies most.

of control is crucial in achieving these Pareto-fronts.

Dimensioning of PV: In Figures 5 and 7, we study the impact of varying PV and EES sizes on mutual information and financial cost. We find that all points at the Pareto-front use the smallest PV size, PV 1. Larger PV sizes do not reduce the mutual information as the EES looses flexibility in storing grid power to flatten the profile. From the information-theoretic perspective on the similarity of two random processes, more mutual information exists between a profile selling PV power to the grid and the original one than a flatter profile and the original one. In future work, instead of either storing or selling PV power, a third option could be to not use excess PV power. From Figure 5, no clear impact of PV sizes on the financial cost is visible. With larger PV sizes, more energy can be sold to the grid, but the cost of purchasing the $\mathrm{PV}$ is also higher.

Dimensioning of EES and quantification of costs: We find that, if the best control is performed, all EES and PV sizes achieve good financial costs while larger EESs result in better (lower) mutual information values as more energy can be stored and hence, a flattening of the grid power is easier to achieve. Combinations of PV 1 with EES 1 and 2 achieve good trade-offs when an appropriate controller is used. The annual costs for the Pareto results of PV 1 with EES 2, as shown in Table III, are about 600 US\$ (data point 12) for a financial cost focused strategy, and 1700 US\$ (data point 13) for a privacy focused strategy. The costs of trade-off strategies naturally lie in between. E.g., the more balanced scheme of data point 11 results in much lower costs of 1000 US\$. Note that the cost for the smallest EES 1 is lowest for a purely aging focused strategy if PV 1 and 2 are installed. This means that the EES cost cannot be compensated by grid gains. For all other cases, the lowest total cost occurs for a grid price focused strategy.

We now analyze the contribution of the grid, PV and aging cost to the total cost. Figure 6 shows a cost breakdown for EES 2 and PV 1 . As expected, the PV cost is the same for all controllers as it only depends on the PV size. The EES aging cost is strongly dependent on the controller, where high aging cost comes with a reduction in mutual information and higher grid cost. The accumulated grid cost from buying and selling 


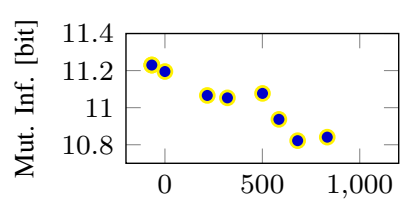

(a) Overhead EES 1, PV 1 (\$)

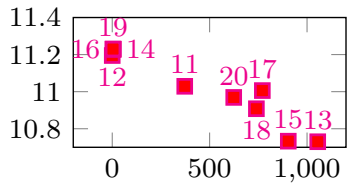

(b) Overhead EES 2, PV 1 (\$)
Fig. 7. Cost overhead: total ownership cost reduced by grid strategy cost. Max. privacy for existing EES and PV costs a) 750 US $\$$ and b) 900 US\$. The node labels in (b) link to the indices in Table III

to the grid varies less in comparison to the EES aging cost. Given the high impact of the aging cost, it may be tempting to assign a high weight to $w_{h}$. However, purely aging optimal strategies, $w_{h}=1$, are not advisable for real employment as they result in keeping the EES at a low SOC without charging or discharging. Adding some aging-awareness results in better cycle life and hence minimal long-term financial losses.

Cost overhead for existing installations: Assuming that owners of already existing EES and PV systems desire to switch from a purely grid optimal strategy to one that improves their privacy, the cost overhead for EES 1,2 with PV 1 is shown in Figure 7. The overhead is determined by the total cost of ownership (aging + grid + PV cost) reduced by the cost of a grid optimal strategy $\left(w_{g}=1\right)$. In few cases, the aging optimal strategy may be cheaper than the grid optimal one resulting in a negative cost overhead. However, a purely aging-focused strategy does not use the EES which can be considered as being poorly dimensioned EES and PV combinations and the respective data points may be ignored. Again, the privacy/cost trade-offs are achieved with appropriate controllers.

Cost splitting: Another interesting analysis our framework provides is whether UPs could offer a special contract to encourage load flattening for users with EES and PV. This is beneficial for the UPs in that they could better manage the electricity grid, and also for the users whose privacy is preserved. However, as indicated from our results, the high battery aging costs result in high costs of flattening. Nevertheless, load flattening could be subsidized by the UP by providing lower rates. Consider the example in Table III The yearly EES, grid and PV costs for a flat profile are approximately 1692 US\$, 682 US\$ and 137 US\$, respectively (data point 13). On the other hand, a financial cost focused strategy entails EES, grid and PV costs of 70 US\$, 430 US\$ and again 137 US\$, respectively (data point 12). Hence, the cost of flattening the profile entails additional aging cost of 1055 US\$ for the consumer. This value is much larger compared to the grid cost, which makes it impractical for the UP to offer a reasonable full compensation scheme. However, in future, when the battery price is expected to drop, the UP could introduce specialized contracts, which motivate consumers to perform load shaving and concurrently protect their privacy.

\section{CONCLUding REMARKS}

Privacy leakage is a serious concern of smart metering systems. However, the installation of an EES and renewable energies can improve privacy while preserving the benefits of smart grids. We present a framework, which allows us to investigate the privacy/cost trade-off. It is based on accurate system models, and provides the most realistic estimations so far. We show how EES and PV size, and also the weights of the cost function impact the privacy, grid cost and aging cost. As for controller design, sensitive data such as PV location and load power histograms are required, future work should also investigate the controller performance under more generic assumptions and the applicability of machine learning. While finding the Pareto-front, we observe that i) proper control of the system is crucial for performance, ii) a strategy solely focusing on privacy results in high financial costs, iii) significant privacy enhancement comes at acceptable but not negligible cost, and iv) when the user increases privacy, the UP benefits from peak shaving and should partially compensate costs.

\section{REFERENCES}

[1] A. Molina-Markham, P. Shenoy, K. Fu, E. Cecchet, and D. Irwin, "Private memoirs of a smart meter," in Proc. of BuildSys, 2010.

[2] P. McDaniel and S. McLaughlin, "Security and privacy challenges in the smart grid," IEEE Security \& Privacy, vol. 7, no. 3, pp. 75-77, 2009

[3] G. Hart, "Nonintrusive appliance load monitoring," Proceedings of the IEEE, vol. 80, no. 12, pp. 1870-1891, 1992.

[4] G. Kalogridis, Z. Fan, and S. Basutkar, "Affordable privacy for home smart meters," in Proc. of ISPAW, 2011.

[5] L. Yang, X. Chen, J. Zhang, and H. Poor, "Cost-effective and privacypreserving energy management for smart meters," IEEE Transactions on Smart Grid, vol. 6, no. 1, pp. 486-495, 2015.

[6] S. Li, A. Khisti, and A. Mahajan, "Privacy-optimal strategies for smart metering systems with a rechargeable battery," in Proc. of ACC, 2016.

[7] G. Giaconi, D. Gündüz, and H. V. Poor, "Smart meter privacy with renewable energy and an energy storage device," IEEE Transactions on Information Forensics and Security, vol. 13, no. 1, pp. 129-142, 2018.

[8] E. Liu and P. Cheng, "Achieving privacy protection using distributed load scheduling: A randomized approach," IEEE Transactions on Smart Grid, vol. 8, no. 5, pp. 2460-2473, 2017.

[9] M. T. Tesfaye, M. Nardello, and D. Brunelli, "Residential electrical consumption disaggregation on a single low-cost meter," in Proc. of EESMS, 2017

[10] A. Zoha, A. Gluhak, M. Imran, and S. Rajasegarar, "Non-intrusive load monitoring approaches for disaggregated energy sensing: A survey," Sensors, vol. 12, no. 12, pp. 16838-16866, 2012.

[11] M. Chen and G. Rincon-Mora, "Accurate electrical battery model capable of predicting runtime and I-V performance," IEEE Transactions on Energy Conversion, vol. 21, no. 2, pp. 504-511, 2006.

[12] G. Suri and S. Onori, "A control-oriented cycle-life model for hybrid electric vehicle lithium-ion batteries," Energy, vol. 96, pp. 644 - 653, 2016.

[13] A. Bocca, A. Sassone, D. Shin, A. Macii, E. Macii, and M. Poncino, "An equation-based battery cycle life model for various battery chemistries," in Proc. of VLSI-SoC, 2015.

[14] U. of Massachusetts Amherst, "Umass smart* home data set," http:// traces.cs.umass.edu/index.php/Smart/Smart 2017.

[15] J. Ehnberg and M. Bollen, "Simulation of global solar radiation based on cloud observations," ISES Solar Energy, vol. 78, no. 2, pp. 157 162, 2005.

[16] S. Barker, A. Mishra, D. Irwin, E. Cecchet, P. Shenoy, and J. Albrecht, "Smart*: An open data set and tools for enabling research in sustainable homes," SustKDD, vol. 111, no. 112, p. 108, 2012.

[17] D. Sera, R. Teodorescu, and P. Rodriguez, "PV panel model based on datasheet values," in Proc. of IEEE-ISIE, 2007.

[18] D. Feldman, G. Barbose, R. Margolis, R. Wiser, N. Darghouth, and A. Goodrich, "Photovoltaic (pv) pricing trends: historical, recent, and near-term projections," NREL, LBNL (United States), Tech. Rep., 2012.

[19] D. C. Jordan and S. R. Kurtz, "Photovoltaic degradation ratesan analytical review," Progress in photovoltaics: Research and Applications, vol. 21, no. 1, pp. 12-29, 2013.

[20] Los Angeles Department of Water and Power, "Electric rate summary," https://www.ladwp.com 2017.

[21] I. Chades, G. Chapron, M. Cros, F. Garcia, and S. R., "Mdptoolbox: a multi-platform toolbox to solve stochastic dynamic programming problems," Ecography, vol. 37, pp. 916-920, 2014.

[22] M. L. Puterman, Markov decision processes: discrete stochastic dynamic programming. John Wiley \& Sons, 2009.

[23] Tesla, "Powerwall: Technical specs," https://www.tesla.com/powerwall 2017. 